\title{
u-track 3D: measuring and interrogating intracellular dynamics in three dimensions.
}

Philippe Roudot*1,4, Wesley R. Legant ${ }^{2,3}$, Qiongjing Zou ${ }^{1,4}$, Kevin M. Dean ${ }^{4}$, Erik S. Welf ${ }^{1,4}$, Ana F. David ${ }^{5}$, Daniel W. Gerlich ${ }^{5}$, Reto Fiolka ${ }^{4}$, Eric Betzig ${ }^{6}$ and Gaudenz Danuser*1,4

1.Lyda Hill Department of Bioinformatics, UT Southwestern Medical Center. Dallas, Texas, U.S.A.

2.Department of Biomedical Engineering, University of North Carolina and North Carolina State University, Chapel Hill and Raleigh, NC, U.S.A.

3.Department of Pharmacology, University of North Carolina, Chapel Hill, NC, U.S.A.

4.Department of Cell Biology, UT Southwestern Medical Center. Dallas. Texas, U.S.A.

5.Institute of Molecular Biotechnology of the Austrian Academy of Sciences. Vienna, Austria.

6.Department of Molecular \& Cell Biology, University of California. Berkeley, California, U.S.A.

* Corresponding Authors: philippe.roudot@utsouthwestern.edu, gaudenz.danuser@utsouthwestern.edu

\section{Abstract}

Particle tracking is a ubiquitous task in the study of dynamic molecular and cellular processes by live microscopy. Light-sheet microscopy has recently opened a path to acquiring complete cell volumes for investigation in 3-dimensions (3D). However, hypothesis formulation and quantitative analysis have remained difficult due to fundamental challenges in the visualization and the verification of large sets of 3D particle trajectories. Here we describe u-track 3D, a software package that addresses these two challenges with three algorithmic innovations. Building on the established framework of globally optimal particle association in space and time implemented in the u-track package and recent advances in gaining association robustness in the case of erratic motion, we first report a complete and versatile pipeline for particle tracking. We then present the concept of dynamic region of interest (dynROI), which allows an experimenter to interact with dynamic 3D processes in 2D views amenable to visual inspection. Third, we present an estimator of trackability, which provides for every trajectory a confidence score, thereby overcoming the challenges of visual validation of trajectories in dense particle 
fields. With these combined strategies, u-track 3D provides a framework for the unbiased study of molecular processes in complex volumetric sequences.

\section{Main}

Light-sheet fluorescence microscopy (LSFM) achieves three-dimensional (3D) imaging with minimal phototoxicity, fast sampling, and near-isotropic resolution ${ }^{1,2}$. Given these advances, many dynamic intracellular processes that were once challenging to study even in $2 \mathrm{D}$ (e.g., mitosis, cytoskeleton organization, vesicle trafficking, molecular interactions), can now be monitored in the entire cellular volume. While the application of computer vision techniques are well established for interrogating cell biological processes in 2D microscopy ${ }^{3}$, these tools do not provide a solution toward the interpretation of dense arrangements of structures moving in a dimensionally unconstrained manner. In 3D, both the visualization of measurement results and their validation require a new set of computational tools. In addition, a key challenge for image analysis in 3D is the limited ability for a user to interact with the data. The manipulation of time-lapse 3D image volumes is often cumbersome, and any of the projection mechanisms necessary to map the 3D volume into a $2 \mathrm{D}$ data representation on a screen is prone with artifacts that may cause false conclusions ${ }^{4}$. Thus, computational tools for 3D image analysis must be able to reveal the complexity of 3D cellular and sub-cellular processes, while being as minimally supervised as possible to avoid selection and perception biases.

The most elementary way to measure the behavior of intracellular processes in space and time is particle tracking. Particles can comprise sub-diffraction sized objects that appear in the image volume as bona fide spots, objects of an extended size that appear as a rigid structure, and larger deformable objects. The more complex the object's shape is, the more sophisticated the methods for particle detection are needed to extract a coordinate triplet $[x(t), y(t), z(t)]$ for each object and for each time 
point. The problem of particle tracking is then defined as the reconstruction of a set of trajectories across time points given the coordinates of the identified particles in the individual time points. Many approaches have been proposed to solve this problem in a computationally feasible manner ${ }^{5-8}$. However, only a few of these methods have been implemented in 3D and none of those approaches tackle the visualization and interpretation challenges ${ }^{5,9-11}$.

Building upon our previous particle tracking work ${ }^{2,7,12}$, we designed the software package u-track 3D to enable the measurement, observation and interrogation of dynamic processes in 3D. u-track 3D can detect and track morphologically and dynamically diverse cellular structures, including single molecules, adhesion complexes, and larger macromolecular structures such as +TIP protein complexes associated with growing microtubules. The software design is open, allowing users to import the coordinate files from other detection routines and then apply the u-track framework only for trajectory reconstruction. To overcome challenges inherent to volumetric data, we introduce an extensive library for visualization and mapping of dynamic region of interests (dynROIs) that move with the biological structure under evaluation and enable an intuitive visualization of particle behaviors. Finally, as it is generally impossible to visually validate the trajectory reconstruction in $3 \mathrm{D}$, we present an algorithm for the automatic assessment of particle trackability throughout the image volume.

To generate a robust 3D particle tracking package, we adopted and modified features that were critical for accurate particle tracking in $2 D^{7}$. This includes the break-down of trajectory reconstruction into a frame-by-frame association of corresponding particles followed by an association of the resulting track segments into full-length trajectories. Both optimization steps rely on the same solution for optimal oneto-one assignments of particle detections and track segments in a bipartite graph ${ }^{7,13}$. The two-step approach permits the closing of temporal gaps in the detection of a particle, as well as the handling of 
particle merging and splitting events that are inherent to many biological processes. As such, the resulting set of trajectory is a global optimum in space and time for a given set of detections. Moreover, u-track 3D incorporates a Kalman filtering approach to model on the fly the characteristics of a particle's Brownian, directed, and heterogeneous motion, which supports both the procedure for frame-by-frame particle association and the one of track segment association.

To assess the performance of u-track 3D, we investigated the dynamics of various cellular structures imaged by light sheet microscopy (Figure 1). As reported with u-track, gap closing is a crucial step in 2D particle tracking because of the frequent transient disappearances: particles might not be detected, particles move in and out of the microscope's in-focus volume, or particles can temporarily overlap in space. While the latter two sources of disappearance are largely eliminated by proper 3D imaging, the challenges of false or missing detections remain. To test the performance of u-track 3D in closing gaps, we examined the lifetimes of clathrin-coated structures forming at the cell plasma membrane (Fig. 1a). These structures represent mostly sub-diffraction objects, i.e. they appear in a 3D imaging volume as point-spread functions. We used high-resolution diagonally swept light-sheet microscopy ${ }^{2}$ to sample every second a 3D image volume of puncta generated by the GFP-labeled AP2 subunit of the endocytic coat. In this volumetric sequence, u-track 3D recovered the canonical lifetime distributions of abortive and maturing clathrin-coated pits, that is an exponential decay for abortive pits and Rayleigh-like distribution with maximal probability around 20 s for maturing pits (Fig. 1.c, Movie 1 and Supplementary Note 1). While the identification of those two populations, has required extensive trajectory analysis to discount incomplete trajectories caused by the limitations of 2D microscopy (e.g., the transient arrival of golgi-associated clathrin coated structures into the evanescent illumination field) ${ }^{14}$, our u-track software achieves accurate trajectory classification directly by thresholding the maximum intensity of trajectories in 3D (Fig 1.b,c). Importantly, the separation of lifetime distributions into two pit classes can 
only be obtained with the support of gap closing (Fig 1.c), suggesting that gaps present a hurdle for accurate tracking also in 3D.

With limited sampling frequency in volumetric imaging due to the serial acquisition of a z-stack comprising tens to hundreds of focal slices, the automated reconstruction of particle trajectories can be improved by dynamic motion models through Kalman filtering. To assess the performance of a 3D implementation of previously published motion models for 2D tracking of microtubule polymerization dynamics $^{15,16}$, we imaged and tracked the dynamics of microtubules in HeLa cells following GFP-fusions of the microtubule plus-end tracking protein EB1 using lattice light-sheet imaging ${ }^{1}$ at $1 \mathrm{~Hz}$ in interphase and metaphase. We quantified metrics such as growth rate, growth lifetime and pause frequency (see Supplementary Note 2). The latter is a measure for the probability that a stalled or shrinking microtubule, which is accompanied by disappearance of the EB1 particle in the movie, is rescued to renewed growth (see Supplementary Figure 1 and Movie 2). Consistent with our previous observations in $2 D^{15}$, u-track 3D faithfully detected a dose-dependent decrease in all three metrics upon treatment of cells with the microtubule-destabilizing drug nocodazole (Fig 1.d-e). We also investigated the destabilizing effect of nocodazole by measuring the number and duration of pauses or shrinkages that occur with drug treatment (Fig 1.e) until disappearance at the highest concentration. We then extended our analyses to mitotic cells, where the density of EB1 particles is much higher in central regions of the mitotic spindle (see Movie 3). Both scenarios show a strong response in nocodazole concentration, indicating that u-track 3D properly captures the drug-induced variation of growth rate and lifetime (Fig 1.f-g), despite strong variations in particle density.

We then sought to investigate the impact of the depth information on the measurement of biological quantities when compared to 2D particle tracking. We compared the u-track gap closing and motion 
modeling capacities, including an approach to follow particle trajectories with erratic go-stop-go behaviors ${ }^{12}$, in both 2D and 3D data using single molecule imaging. We used lattice light-sheet to image the interactions between transcription factors (TFs) and chromatin in embryonic stem cells. In a study using the same biological system, Chen et $\left.a\right|^{17}$ had shown that TFs alternate between short-lived binding events at non-specific chromatin sites (residence time $\sim 0.75 \mathrm{~s}$ ), 3D diffusion (average duration $\sim 3 \mathrm{~s}$ ) and longer lived transcription events where the TF is bound at specific chromatin sites (residence time $12 \mathrm{~s}$ ). These results were quantified in $2 \mathrm{D}$ using both light-sheet and widefield imaging. We performed the same analysis now applying 3D tracking and contrasted the results to the tracking of 2D projections of the same 3D volumes (Fig 1.h-k and Supplementary Note 3). Interestingly, when analyzed in 3D, the residence time of binding events was reduced by one third ( 7.8s in 3D vs $11.9 \mathrm{~s}$ in $2 \mathrm{D})$. Thus, we are only able to reproduce the results quantified in the original study on 2D projections, which differ significantly from the 3D results. Interestingly, the shorter binding time observed in 3D trajectories is consistent with measurements performed in nuclear receptors studies ${ }^{18,19}$. Together, these data suggest that the overlap caused by axial integration of the fluorescent signal imaged in 2D may artificially prolong the lifetimes and change the conclusion on binding kinetics.

Moving from 2D to 3D images complicates the interaction of a human observer with both raw and derived data. Widely used global image projections, including maximum intensity projection, and other volume rendering techniques are limited by the overlap of many dynamic structures along the viewing axis $^{4}$. However, detailed visualization of 3D images and trajectories in their local context is essential for a user to adjust software control parameters and to interpret the underlying biology. $t$ As such, projection approaches have to be tailored to emphasize a subset of selected voxel or aspects of highest interest. Such projections should not only bring the particle or group of particles of interest into focus, but also continuously adapt as the particles move. To meet this requirement, u-track 3D incorporates a 
framework for rendering particle-centric dynamic regions of interest (dynROIs), thereby allowing the user to follow the particle behavior throughout its lifetime in a visually-comprehensible format.

DynROls are implemented in a hierarchical object structure across molecular, macromolecular and cellular scales. The top level dynROI is defined for the cell. For example, cells embedded in a 3D environment are often randomly oriented, and their orientation changes throughout time (Fig 2.a and Movie 4). While image-based registration can be used to correct changes in cell orientation, it is computationally expensive, especially as the size of the volume and length of the sequence grows. To reduce the computational burden, we segment and transform the cell mask into a randomly downsampled point cloud, which is then used to estimate an affine transform between consecutive time points (Fig. 2b and Supplementary Note 5). We illustrate the application of a whole cell dynROI with the study of spatial interactions between cell-matrix adhesions and fluorescently labeled 3D collagen fibers in osteosarcoma cells imaged by axially swept light-sheet microscopy ${ }^{20}$. The dynROI allowed us to visualize the relationship between adhesion shapes and its proximity to collagen fibrils, showing two populations of globular and elongated adhesions (Fig $2 \mathrm{~b}-\mathrm{d}$ ). The most elongated adhesions are located primarily at the tip of the pseudopodial extensions and align with the protrusive direction, while the round adhesions concentrate in the quiescent part of the membrane. Our measurements show that this elongation distribution can be decomposed further (Fig 2e). We found a unimodal distribution of primarily globular adhesions in close contact with collagen fibers, assessed by a score that integrates the distances between voxels in adhesions and collagen fiber masks (see Supplementary Note 6). In contrast, adhesions with a lesser degree of collagen contact display a bimodal distribution of globular and elongated adhesions. These data suggest - quite unexpectedly - that unlike in 2D the most engaged adhesions are the least elongated ${ }^{21}$. We conjecture from this finding that adhesion elongation in $3 D$ is less driven by a zippering of an integrin-mediated plaque along a collagen fiber, but rather is 
dictated by the organization of cell-cortical actin fibers or the local collagen architecture. Indeed, this behavior becomes apparent by replay of time-lapse sequences of the proximity and elongation parameters in the spatially stabilized dynROI (Movies 5, 6 and 7). DynROls are thus a powerful way to assess the spatial distribution and heterogeneity of molecular interactions in highly dynamic cells.

Many cellular processes involve a massive reorganization of multiple macromolecular structures, which challenges 3D analysis by conventional visualization approaches. A classic example is the mitotic spindle of vertebrate cells ${ }^{22}$. While thousands of microtubules reorganize to form a dense bipolar array, the two spindle poles move apart and rotate back and forth. At the same time, spindle microtubules establish contacts with chromosomes at specialized attachment sites termed kinetochores, and subsequently move chromosomes towards poles or the spindle center. The complexity of these structures and their rapid dynamics are virtually impossible to understand by mere visual inspection of volume renderings. We therefore assessed how u-track 3D and dynROIs facilitates the analysis of this process. The image dataset comprises dual-channel time-lapse sequences of GFP-labelled microtubule plus-ends and mCherry-labelled kinetochores of mitotic HeLa cells, acquired by lattice light sheet microscopy as described in ref $^{23}$, with an acquisition frequency of $0.1 \mathrm{~Hz}$ to enable longer imaging, from prometaphase to metaphase. To identify and track the dynamics of various spindle components, we devised a multiscale particle detector designed to identify particles of various sizes and contrast (see Supplementary Note 7). In addition to microtubule plus-ends and kinetochores, our multiscale approach is able to detect spindle poles based on the diffuse clouds of microtubule plus-end marker. Pole trajectories can then be used to define a dynROI that follows the spindle motion (Fig $2 . f$ and Supplementary Note 5.3). An embedded second dynROI follows the point cloud formed by the kinetochore trajectories (Fig. 2.g and Supplementary Note 5.4). The progression of these dynROIs over time is determined by the same point cloud tracking approach as described above for the definition of a 
whole cell dynROI. Based on the pair of dynROIs, we further construct a planar dynROI with an orientation that is defined by the interpolar axis and a vector following the kinetochore-associated dynROI motion (Fig 2.h,i, movie 8, 9 and Supplementary Note 5.5). Our framework for dynROI estimation thus enables the automated visualization of dynamic mesoscale structures composed of different molecular assemblies.

The hierarchical model of dynROIs also enables the detailed analysis of microscale collective molecular processes that move throughout the cellular volume. This facilitates the study of complex subprocesses, as for example the formation of kinetochore fibers during spindle assembly. In previous work ${ }^{23}$, we showed with spindle-wide statistics and indirect modeling that kinetochore fiber formation is accelerated by an augmin-dependent nucleation and directional growth along the fiber towards kinetochores. To expand the analysis of this process, we now use dynROIs to directly visualize the dynamic space between spindle poles and kinetochores (Fig. 2j-I and Movie 10). We define a kinetochore fiber assembly dynROI by a cone whose medial axis connects spindle pole and a target kinetochore (see Supplementary notes 5.6). By systematically inspecting kinetochore fiber assembly dynROIs, we observed a strong directional bias in microtubule polymerization along a kinetochore fibers and toward kinetochores, consistent with previous observations ${ }^{23}$. Occasionally, we also observed microtubule polymerization branching off from a kinetochore fiber and polymerizing toward another kinetochore (circled in red in Fig. 2.k,l, time $53 s-72 s$ ). The branching was followed by a rapid poleward motion of the targeted kinetochore and an increase of plus-end count in the dynROI (Fig. $2.1, m$, time $93 \mathrm{~s}-102 \mathrm{~s}$ ) suggesting that the target kinetochore was captured, and that this new capture established a new avenue for microtubule amplification. This data suggests that kinetochore capture might occasionally involve interactions between neighboring kinetochore fibers. This example 
underscores how the dynROI library implemented in u-track 3D enables the visual discovery of dynamic processes that are completely obscured in 3D image volumes.

Validation of tracking results is crucial for proper parameter adjustment of image acquisition and analysis, and for the biological interpretation of integrated measurements. However, it remains an extremely challenging task in 3D datasets, particularly when the particle density is high. Contrary to a scenario in 2D where a single field of view presents a wide range of trajectories for visual inspection, dynROIs in 3D tend to capture only a few trajectories and cannot represent the heterogeneity of local image quality, particle density and dynamic properties, which all affect the tracking accuracy. To solve this problem, we complement u-track 3D with the computation of a local trackability score. For a scenario of homogeneous particle density and directionally random displacements, we offered in previous work a model to compute the probability of tracking errors ${ }^{2}$. However, in a more realistic model, each trajectory bears its own level of uncertainty based on its own stochastic footprint and the local configuration of particle locations (Fig 3.a). Here, we compute for every trajectory and every time point the confidence by which the algorithm was able to assign the chosen particle to the trajectory (see Supplementary Note 8). Specifically, we exploit the particle history, the detection accuracy and the associated motion model(s) to derive a trackability metric that represents the likelihood of each of the chosen associations vis-à-vis the set of alternative associations with neighboring particles. Fig 3.a shows an example of ambiguous associations where two assignment hypotheses between detections and track segment heads have a similar association cost. Despite this ambiguity, the linear programming approach identifies a single optimal solution without the possibility for error estimation. To determine the level of ambiguity, we resample all track head predictions in frame $t$ and test the stability of the original assignment in frame $t+1$. The approach is illustrated in Fig 3.b-d based on the tracking of transcription factor in a dataset acquired with multifocus microscopy and previously published in ref $^{24}$ (see 
Supplementary Note 4). Each dot describes a sample and the color of each sample indicates whether the newly computed assignment matches the original, or points to another particle or track segment termination. The trackability score is defined as the fraction of tested assignments matching the original assignment (Fig 3.e). Importantly, the trackability score is not a deterministic identifier of tracking errors. Links with a high score can still be false and links with a low score can still be true.

We first tested the capacity of the trackability score to describe variations in tracking quality in space and time on the trajectories of endocytic pits (see Supplementary Note 9). The cells were plated on collagen and imaged using high-resolution diagonally swept light-sheet microscopy ${ }^{2}$. The acquisitions present a large variety of dynamic behaviors, from a quiescent membrane in the center to rapid protrusive displacements at the leading edge. To selectively analyze those areas, we manually selected three dynROIs to reflect a quiescent area, a slow protrusion-retraction cycle and a fast protrusionretraction cycle. To account for sample motion, those dynROIs were selected within a larger dynROI built from all the trajectories detected (Movie 11). The trackability score shows a consistently high score in the quiescent dynROI, a large and transient decrease in trackability around the fast protrusive motion and an average decrease in the slower protrusion (Fig 3.e-g). Our score is thus able to accurately reflect the spatiotemporal variation in trackability and detect the time points when ambiguity arises due to rapid protrusive motions.

In a second experiment we tested the capacity of our trackability score to compare tracking quality in a complex scene with heterogeneous cellular structures. We analyzed the spindle assembly dataset with labelled microtubule plus-ends and kinetochores. The cumulative distributions of trajectory-averaged trackability scores showed that kinetochore trajectories are more reliably detected compared to microtubule plus-end trajectories (Fig. 3.i), consistent with their much lower density and slower motion. 
Our score enables the analysis of trackability on a per-trajectory basis. Indeed, selecting the 90th percentile of trackability score reveals the locations of error-free trajectories for both plus-end and kinetochore channels (Fig. 3.j). In contrast, the $10^{\text {th }}$ percentile of the trackability score distribution for plus-end trajectories picks out an erroneous trajectory surrounded by a dense population of other microtubules polymerizing in heterogeneous directions. Because of the overall much higher trackability of the kinetochore channel, the $10^{\text {th }}$ percentile of the trackability score distribution of these trajectories picks out a trajectory with only one association error related to a false positive particle detection. The trackability score can thus be used to assess the overall accuracy of tracking results in a given imaging channel, and it further assists selection of correctly tracked objects in a dense population of trajectories.

In summary, we report here a new version of the popular tracking framework u-track, which now enables the study of particle dynamics in 3D live microscopy. First, we demonstrate the 3D implementation of the global optimization of particle trajectory reconstruction in space and time, including several particle motion models that support particle tracking in a variety of imaging and biological scenarios. We demonstrate applications of the u-track 3D software to the measurement of lifetimes of endocytic events, of microtubule growth dynamics, and of the stop-and-go behavior of individual transcription factors binding to chromatin. We introduce dynROIs, which unveil local particle behaviors embedded in highly dynamic sub-cellular processes that would otherwise be buried in global renderings of the 3D trajectories. We illustrate these functionalities across scales, from the cell-wide heterogeneity of adhesions in a collagen-embedded cell, to the microscale organization of microtubules during chromosome capture. Finally, we introduce a strategy for automatic detection of tracking ambiguities that pinpoint the locations and times of potential tracking errors in the full set of reconstructed trajectories. We demonstrate the approach on broad applications, from mapping of the spatial heterogeneity of tracking quality in the quantification of endocytic events, to the automatic 
identification of high and low quality trajectories imaged in the mitotic spindle. Thus, u-track 3D complements the revolution in 3D live imaging by light-sheet microscopy with a tool set for the unsupervised and supervised quantitative analysis the biological processes these movies capture.

\section{Authors contributions}

P.R. and G.D. designed the research. P.R. wrote the tracking and rendering software, and performed image analysis. Q.Z. developed the software's graphical user interface. W.R.L., K.M.D., E.S.W. and A.D. performed the biochemical and imaging experiments. K.M.D., R.F. and E.B. provided imaging resources. P.R. and G.D. wrote the manuscript. All authors read and provided feedback on the final manuscript.

\section{Acknowledgments}

The authors are grateful to Yuko Mimori-Kyosue at the RIKEN Institute for the gift of the HeLa cells expressing eGFP-EB1 and fruitful conversations. We are also grateful to Zhe Liu at Janelia Research Campus for giving the E.S. cells. P.R. has been funded by the fellowship LT000954/2015 from the Human Frontiers in Science Program. Work in the Danuser lab has funded by the grant R35GM136428. W.R.L. acknowledges support from the Searle Scholars Program, the Beckman Young Investigator Program, an NIH New Innovator Award (DP2GM136653) and the Packard Fellows Program. K.M.D receives partial salary support from R01MH120131, R34NS121873, and R01DK127589. Research in the laboratory of D.W.G. has been supported by the Vienna Science and Technology Fund (WWTF; project nr. LS14-009), and by the Austrian Science Fund (FWF special research program SFB "Chromosome Dynamics"; project nr. SFB F34-06). R.F. has been supported by the Cancer Prevention Research Institute of Texas (RR160057), and the NIH (R33CA235254 and R35GM133522). Lattice light-sheet imaging of the mitotic spindle data produced in collaboration with the Advanced Imaging Center, a facility jointly supported by 
the Gordon and Betty Moore Foundation and Howard Hughes Medical Institute at the Janelia Research Campus.

\section{References}

1. Chen, B.-C. et al. Lattice light-sheet microscopy: Imaging molecules to embryos at high spatiotemporal resolution. Science 346, 1257998 (2014).

2. Dean, K. M. et al. Diagonally Scanned Light-Sheet Microscopy for Fast Volumetric Imaging of Adherent Cells. Biophys. J. 110, 1456-1465 (2016).

3. Kervrann, C., Sorzano, C. O. S., Acton, S. T., Olivo-Marin, J. C. \& Unser, M. A Guided Tour of Selected Image Processing and Analysis Methods for Fluorescence and Electron

Microscopy. IEEE J. Sel. Top. Signal Process. 10, 6-30 (2016).

4. Driscoll, M. K. \& Danuser, G. Quantifying Modes of 3D Cell Migration. Trends Cell Biol. 25, 749-759 (2015).

5. Chenouard, N. et al. Objective comparison of particle tracking methods. Nat. Methods 11, $281-289$ (2014).

6. Smal, I. \& Meijering, E. Quantitative comparison of multiframe data association techniques for particle tracking in time-lapse fluorescence microscopy. Med. Image Anal. 24, 163-189 (2015).

7. Jaqaman, K. et al. Robust single-particle tracking in live-cell time-lapse sequences. Nat. Methods 5, 695-702 (2008).

8. Sbalzarini, I. F. \& Koumoutsakos, P. Feature point tracking and trajectory analysis for video imaging in cell biology. J. Struct. Biol. 151, 182-195 (2005). 
9. Godinez, W. J. \& Rohr, K. Tracking virus particles in fluorescence microscopy images via a particle Kalman filter. in Proc. Int. Symp. on Biomedical Imaging (ISBI) 532-535 (2015). doi:10.1109/ISBI.2015.7163928.

10. Wolff, C. et al. Multi-view light-sheet imaging and tracking with the MaMuT software reveals the cell lineage of a direct developing arthropod limb. eLife 7, e34410 (2018).

11. Chenouard, N., Bloch, I. \& Olivo-Marin, J. C. Multiple Hypothesis Tracking for Cluttered Biological Image Sequences. IEEE Trans. Pattern Anal. Mach. Intell. 35, 27363750 (2013).

12. Roudot, P., Ding, L., Jaqaman, K., Kervrann, C. \& Danuser, G. Piecewise-Stationary Motion Modeling and Iterative Smoothing to Track Heterogeneous Particle Motions in Dense Environments. IEEE Trans. Image Process. 26, 5395-5410 (2017).

13. Jonker, R. \& Volgenant, A. A shortest augmenting path algorithm for dense and sparse linear assignment problems. Computing 38, 325-340 (1987).

14. Aguet, F., Antonescu, C. N., Mettlen, M., Schmid, S. L. \& Danuser, G. Advances in Analysis of Low Signal-to-Noise Images Link Dynamin and AP2 to the Functions of an Endocytic Checkpoint. Dev. Cell 26, 279-291 (2013).

15. Matov, A. et al. Analysis of microtubule dynamic instability using a plus-end growth marker. Nat. Methods 7, 761-768 (2010).

16. Applegate, K. T. et al. plusTipTracker: Quantitative image analysis software for the measurement of microtubule dynamics. J. Struct. Biol. 176, 168-184 (2011).

17. Chen, J. et al. Single-Molecule Dynamics of Enhanceosome Assembly in Embryonic Stem Cells. Cell 156, 1274-1285 (2014). 
18. Paakinaho, V. et al. Single-molecule analysis of steroid receptor and cofactor action in living cells. Nat. Commun. 8, 1-14 (2017).

19. Voss, T. C. et al. Dynamic Exchange at Regulatory Elements during Chromatin

Remodeling Underlies Assisted Loading Mechanism. Cell 146, 544-554 (2011).

20. Dean, K. M., Roudot, P., Welf, E. S., Danuser, G. \& Fiolka, R. Deconvolution-free

Subcellular Imaging with Axially Swept Light Sheet Microscopy. Biophys. J. 108, 2807-2815 (2015).

21. Gardel, M. L. et al. Traction stress in focal adhesions correlates biphasically with actin retrograde flow speed. J. Cell Biol. 183, 999-1005 (2008).

22. Heald, R. \& Khodjakov, A. Thirty years of search and capture: The complex simplicity of mitotic spindle assembly. J. Cell Biol. 211, 1103-1111 (2015).

23. David, A. F. et al. Augmin accumulation on long-lived microtubules drives amplification and kinetochore-directed growth. J. Cell Biol. 218, 2150-2168 (2019).

24. Grimm, J. B. et al. Bright photoactivatable fluorophores for single-molecule imaging. Nat. Methods 13, 985-988 (2016). 

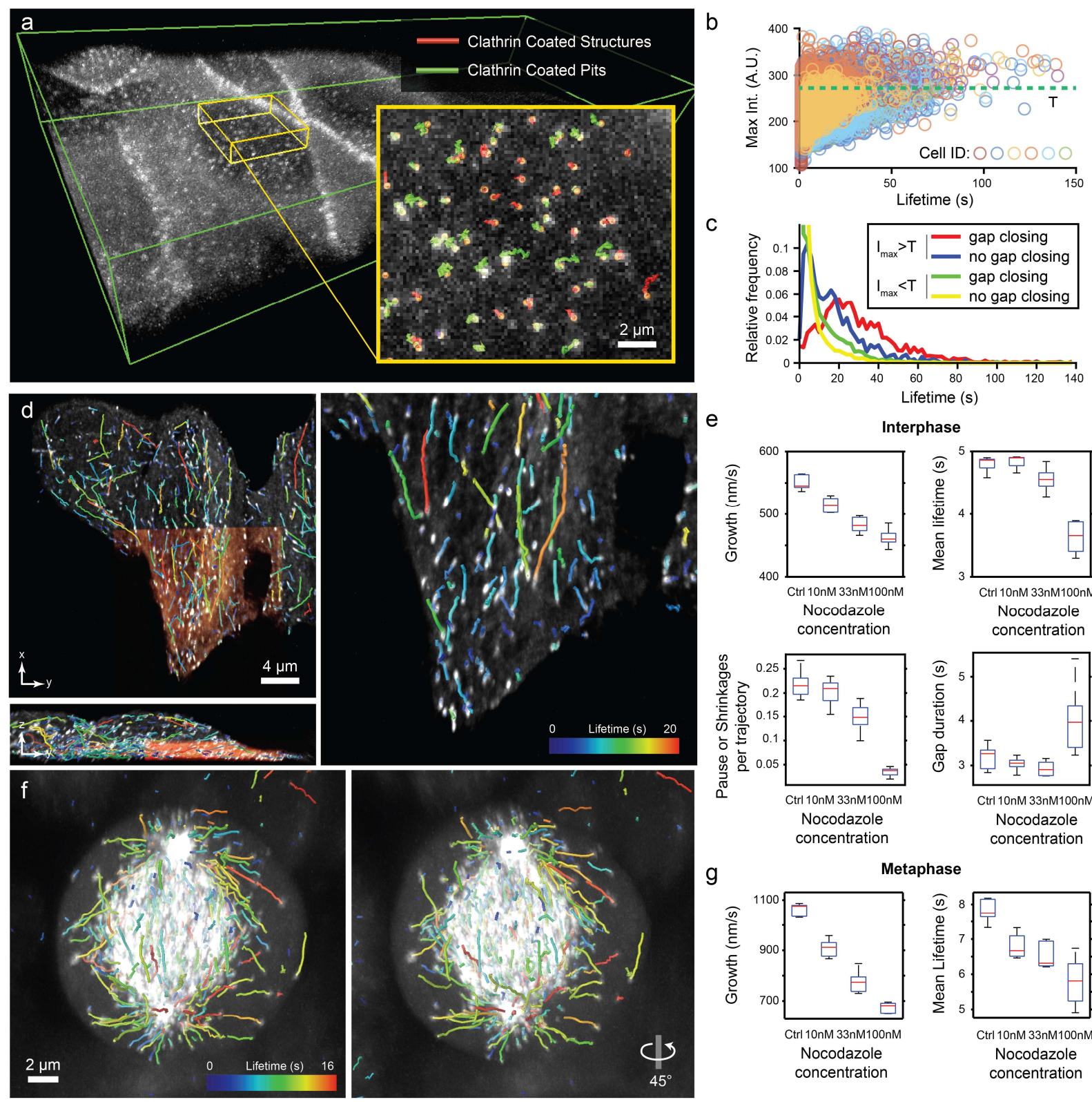

西

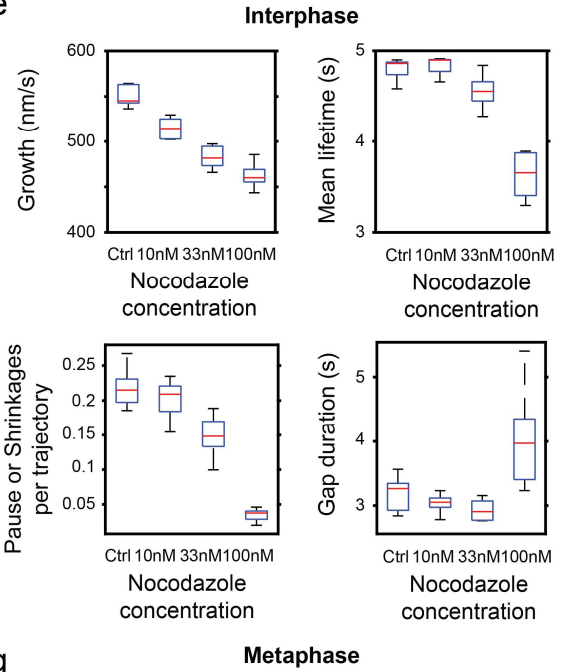

g
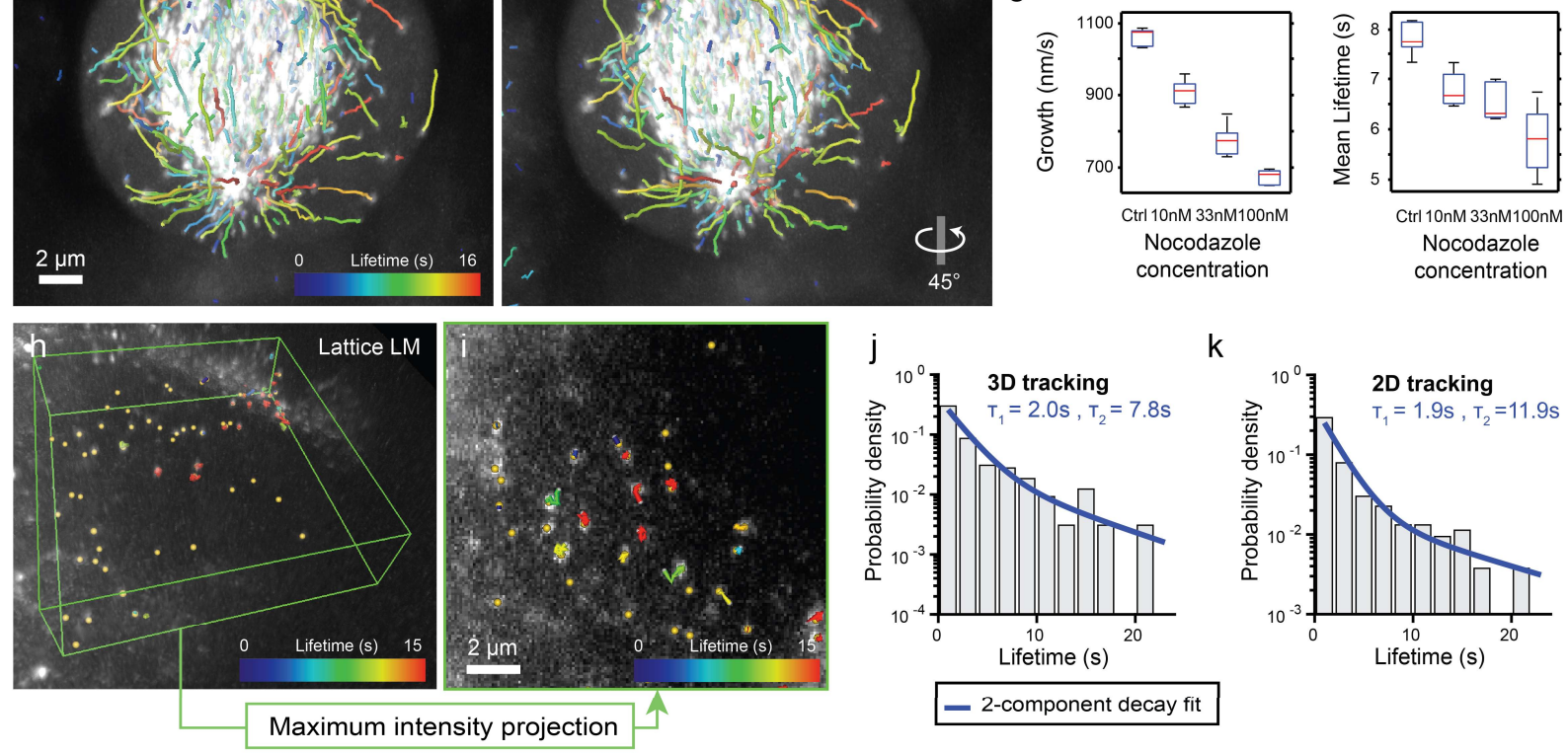

Figure 1: U-track 3D supports a variety of imaging and biological scenarios 
a) Maximum intensity projections (MIP) of a rat kidney cell layer imaged with diagonally scanned light-sheet microscopy (diaSLM). Cells are expressing eGFP-labelled alpha subunit of the AP-2 complex. Green box is 160x40x12 um. Inset shows trajectories of clathrin aggregates classified as clathrin coated structures or maturing pits.

b) Normalized maximum intensity of each trajectory as a function of lifetime plotted for six cellular layers composed of multiple cells each. The green line denotes the median of the cumulated distribution (value T).

c) Probability density of lifetime for the set of trajectories above and below the threshold value T, with and without gap closing.

d) MIP of HeLa cells in interphase imaged with lattice light-sheet microscopy (LLSM) expressing eGFP-labeled EB1 (orange area is $30 \times 32 \times 7 \mathrm{um}$ ). Overlay highlights EB1 trajectories.

e) Average microtubule lifetimes, microtubule growth rate as well as average number and duration of pause and shrinkage events per trajectory for increasing concentrations of nocodazole ( $\mathrm{N}=5$ per conditions).

f) MIP of HeLa cells in metaphase imaged with LLSM along with 45-degree rotation around the vertical axis. Overlay highlights EB1 trajectories.

g) Same as e) measured for cells in metaphase ( $N=5$ per conditions).

h) MIP of mouse embryonic stem cell (ES) nucleus imaged with LLSM expressing GFP-labeled transcription factors. Green box is $13 \times 13 \times 3$ um. Overlay highlights SOX2 trajectories.

i) MIP of ES cell nucleus imaged with LLSM expressing GFP-labeled transcription factors. Overlay highlights SOX2 trajectories tracked after MIP transformation.

j) Probability density of SOX2 binding time measured in LLSM overlaid with a 2-component decay fit.

k) Probability density of SOX2 binding time measured in projected LLSM data overlaid with a 2-component decay fit. 


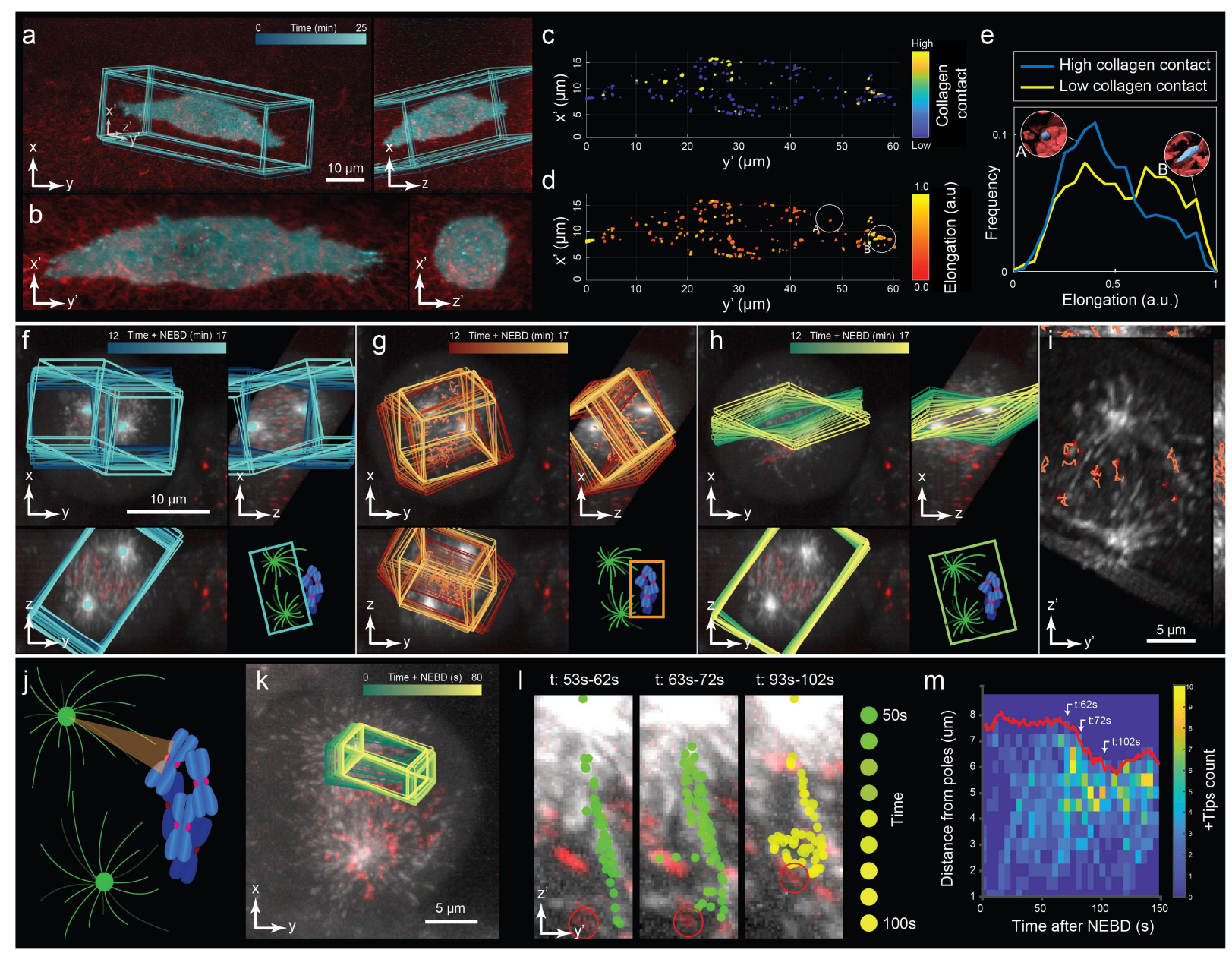

Figure 2: Dynamic Regions of Interest (dynROI) reveals the behavior of local particle involved in highly dynamic sub-cellular processes.

a) Dual-colored orthogonal MIP of osteocarcinoma cells expressing eGFP-labeled paxillin and embedded in collagen labelled with Alexa fluor 568. Overlay highlights dynamic region of interest (dynROI).

b) View of the dynROI.

c) - d) Detection of adhesions colored as a function of the degree of collagen contact and elongation.

e) Probability density of elongation for adhesions with high and low degree of contact with collagen fibers.

f) - h) Dual-colored orthogonal MIP of HeLa cells undergoing mitosis labeled with eGFP-labeled EB3 and mCherrylabeled CENPA. Overlays highlight, f) a dynROI built around centrosome trajectories, g) a dynROI built around CENPA trajectories, and h) a plane built to visualize the dynamics of chromosomes relative to the spindle location. i) View of the dynROI following described in $\mathrm{h}$. 
j) Definition of a conical dynROI between a centrosome and a kinetochore.

k) Dual-colored orthogonal MIP of HeLa cells during pro-metaphase. Overlay highlights the motion of the dynROI.

I) Cumulative overlays of the detected microtubule plus-end position for three periods of 10 seconds between $53 \mathrm{~s}$ to 102 s post nucleus envelope breakage.

m) Plus-ends count function of time and distance from the pole. 
a

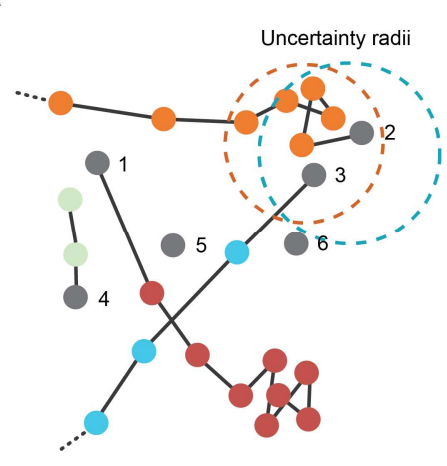

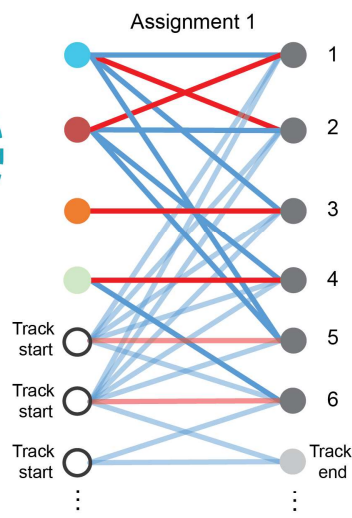

end
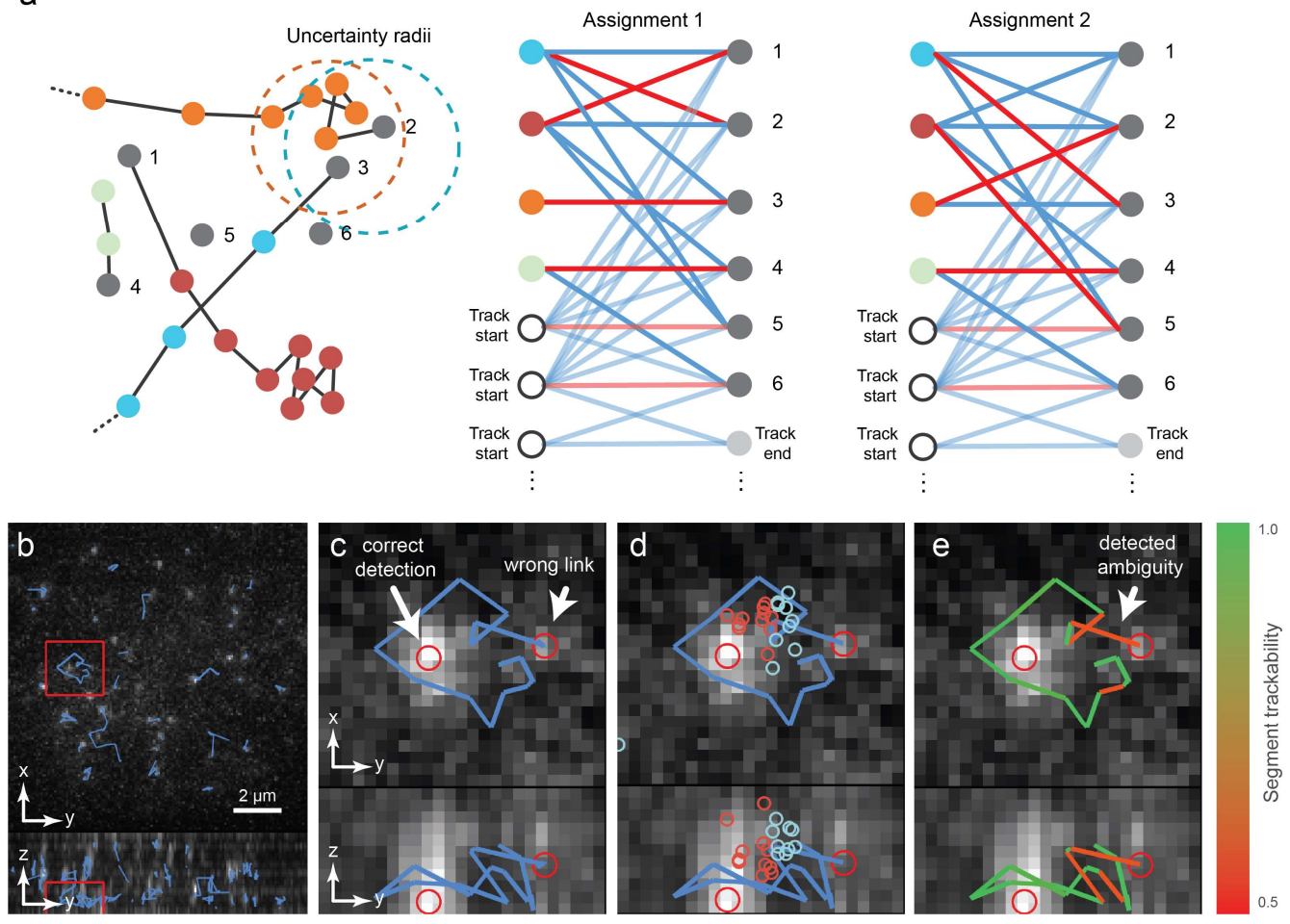

Vote of resampled prediction: Same Different
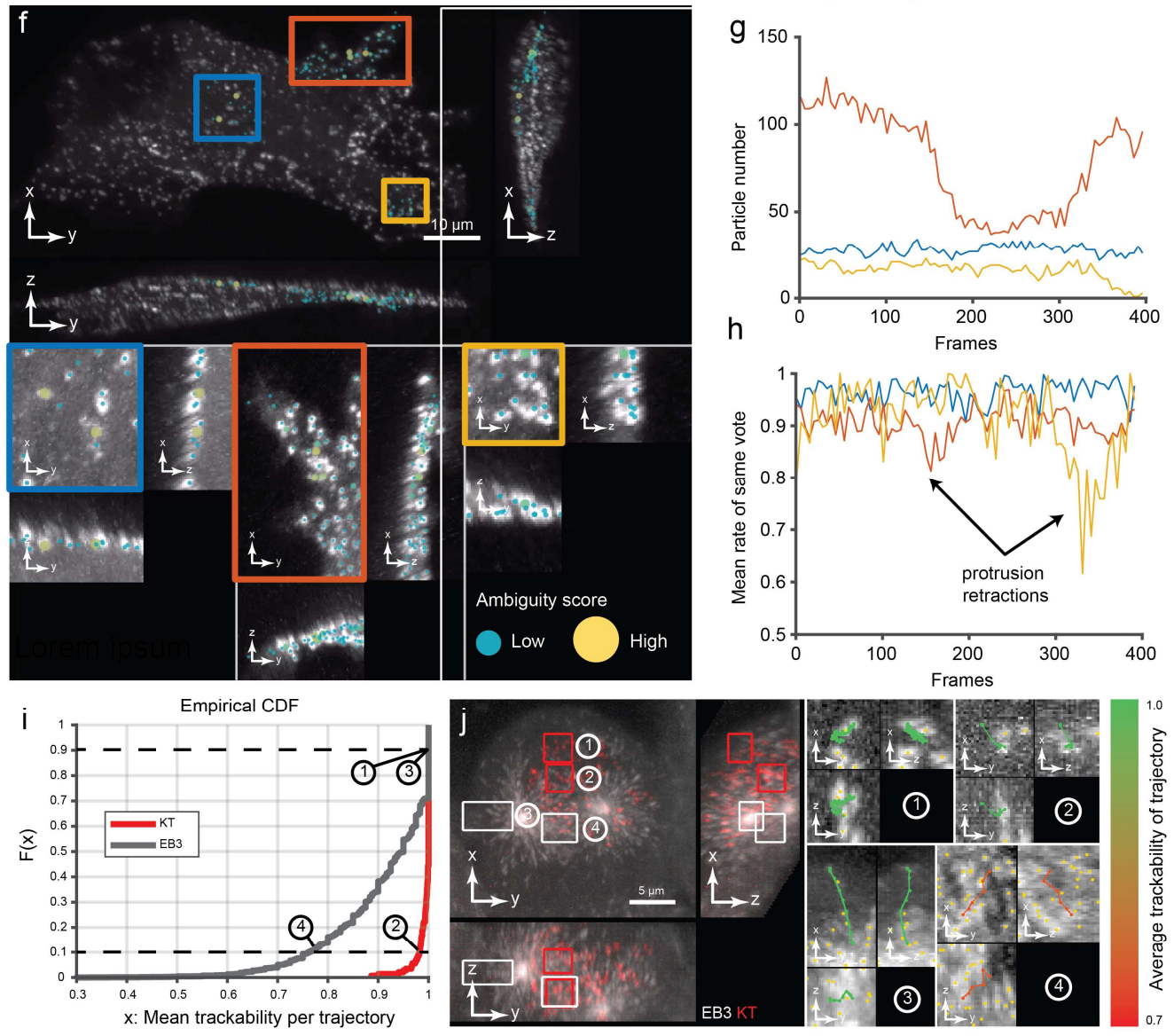
Figure 3: The trackability score pinpoints the locations and times of potential tracking errors across space, time and molecular processes.

a) Diagram showing an example of ambiguous tracking with two possible assignments.

b) Orthogonal MIP of ES cells imaged with MM. Overlays highlight a ROI.

c) Orthogonal MIP of ROI. Overlay shows a trajectory where two close detections create assignment ambiguity

d) Overlay illustrates the stochastic resampling of the predicted position at this time point.

e) Overlay shows trajectory segments colored according to estimated trackability score.

f) Orthogonal MIP of breast cancer cells imaged with diaSLM expressing eGFP-labelled alpha subunit of the AP-2 complex. Rectangular overlays show ROIs with different types of activity. Dot overlays show local level of ambiguity.

g) Number of track segments over time for the three ROIs.

h) Mean rate of stable vote function of time for the three ROIs.

i) Cumulatative distribution of the average trackability score of trajectories for both EB3 and Kinetochore channels.

j) Four ROIs (two for each channel) showing trajectories colored according to their mean trackability. ROI chosen as the $10^{\text {th }}$ and $90^{\text {th }}$ percentiles of the cumulative distribution of trajectory-averaged trackability scores for Kinetochores and EB3 trajectories. Yellow dots show surrounding detections. 